\title{
January 2017 Arizona Thoracic Society Notes
}

The January 2017 Arizona Thoracic Society meeting was held on Wednesday, January 25, 2017 at the HonorHealth Rehabilitation Hospital beginning at 6:30 $\mathrm{PM}$. This was a dinner meeting (prime rib) with case presentations. There was a good attendance representing the pulmonary, critical care, sleep, and radiology communities.

There was a discussion of supporting the Tobacco 21 bill which has been introduced into the Arizona State Legislature. There was unanimous support for this bill. Another bill to allow school nurses to administer an albuterol inhaler without a doctor's prescription was also discussed but the members wanted more information.

The new CDC Ventilator-Associated Events (VAE) criteria were also discussed. Before endorsing or opposing the this as a measure, the members wished more information.

It was decided that a decision on both would be postponed until discussed at the next meeting.

Three cases were presented:

1. Dr. Lewis Wesselius from the Mayo Clinic presented a case of a 53-yearold woman who presented with hemoptysis. The hemoptysis was eventually shown to be secondary to mitral stenosis. There were some dramatic photographs from the bronchoscopy of hyperemic airways with dilated submucosal veins. This case was also presented as the January 2017 Pulmonary Case of the Month in the SWJPCC.

2. Dr. Kyle Henry from Banner University Phoenix/VA presented a case of combined emphysema and pulmonary fibrosis. The pros and cons of establishing a diagnosis were discussed. Although a biopsy would be considered ideal, the patient was severely hypoxemic.

3. Dr. Gerald Swartzberg presented several cases of cavitary coccidioidomycosis. A discussion followed regarding management of cavitary cocci ensued.

There being no further business, the meeting was adjourned about $8 \mathrm{PM}$. The next meeting will be in Phoenix on Wednesday, March 22, 2017 at 6:30 PM at HonorHealth Rehabilitation Hospital.

Lewis J. Wesselius, MD

President, Arizona Thoracic Society 